\title{
10 years of Cell Death \& Disease
}

\author{
Mauro Piacentini ${ }^{1,2}$, Yufang Shi ${ }^{3,4}$ and Hans-Uwe Simon $\mathbb{1}^{5,6}$
}

Cell Death \& Disease is celebrating its 10th anniversary. In the last 10 years from its foundation the content of Cell Death \& Disease has covered an enormous breadth of subjects at the forefront of experimental medicine and clinical practice. The Journal's mission aims to encompass the breadth of translational implications of cell death in the pathogenesis of major human diseases. To mark this important milestone, we requested updates of the research of those scientists who have significantly contributed in this decade to the activity of the Journal with highly cited publications. These high influential original papers together with topical reviews have led Cell Death $\mathcal{E}$ Disease to become a successful landmark in science publishing as highlighted by its growing altmetrics and high impact factor (2019 IF 6.3). The central purpose of Cell Death \& Disease is the publication of original peerreviewed work that constitutes the true basis for advancing biomedical science. This commemorating review set includes four reviews dealing with hot aspects of cancer such as the role cell death regulators, exosomes, metabolism, and long non-coding RNAs and their possible implications in therapy ${ }^{1-4}$. The fifth contribution encompasses the recent developments in the field of metabolic diseases such as non-alcoholic fatty liver disease (NAFLD), the most common cause of chronic liver disease worldwide ${ }^{5}$. One of the major translational achievement of the cell death field is the development of BCL-2 family protein inhibitors such as Venetoclax (ABT199, GDC-0199), the first clinically approved drug of this class, which is currently used in the treatment of chronic lymphocytic leukemia (CLL) as well as acute myeloid leukemia (AML) ${ }^{6}$. However, despite remarkable clinical results, a prolonged treatment with the Venetoclax monotherapy leads to drug resistance. The review by Kapoor et al. ${ }^{1}$ analyzes in detail the mechanism of action of BCL-2 inhibition focusing on the acquired resistance to

Correspondence: Mauro Piacentini (mauro.piacentini@uniroma2.it)

"Department of Biology, University of Rome "Tor Vergata", Rome, Italy

${ }^{2}$ National Institute for infectious Disease IRCCS"Lazzaro Spallanzani", Rome, Italy

Full list of author information is available at the end of the article venetoclax as well as on the possibility that tumors initially resistant to Venetoclax become responsive to it following prior therapies. In the last few years, we have also witnessed another revolution in cancer treatment through targeting the tumor microenvironment to enhance the antitumor immunity. The cytotoxic T-lymphocyteassociated antigen 4 (CTLA-4) and programmed death 1 (PD-1) immune checkpoints are negative regulators of T-cell immune function and their inhibition resulting in increased activation of the immune system is the new frontiers in cancer treatment ${ }^{6}$. However, many cancer patients do not respond initially or develop secondary resistance. In their review, Cerezo and Rocchi ${ }^{2}$ discuss how targeting metabolism could help modulate antitumor immunity and exploring the possibility that the metabolic reprogramming in cancer cells may represent a way to favor the antitumor response ${ }^{7}$. Another key factor in the development of cancer tumor resistance to therapy is discussed in the review by Dong et al. $^{3}$ highlighting the contribution of exosomes to drug resistance in breast cancer. The authors dissect out the role of exosomes biogenesis, the influence of their cargos, and the pattern of release in response to drug treatment. In particular, they discuss how proteins or non-coding RNAs contained in exosomes released in the tumor microenvironment by the tumor itself and/or the stromal cells are able to influence drug resistance by altering the metabolism, prosurvival signaling, epithelial-mesenchymal transition, stem-like property of breast cancer. Breast cancer metastasis is the major cause of mortality in female patients ${ }^{8}$. An increasing number of studies published in Cell Death $\mathcal{E}$ Disease focus on the involvement of long non-coding RNAs (lncRNAs) in cancer metastasis; however, a defined role of these IncRNAs are yet to be clarified $^{9-11}$. The review by Liu et al. ${ }^{4}$ focuses on the lncRNAs functions in breast cancer invasion and metastasis, with particular emphasis on their dual functions for metastasis, their functional mechanisms, their regulatory factors, and the therapeutic promises. The last review by Rada et al. ${ }^{5}$ of this collection does not appear to be related to the 
previous ones at a first glance. Indeed it discusses a metabolic aspect that is assuming more and more importance nowadays. In fact, non-alcoholic fatty liver disease (NAFLD) is the commonest cause of chronic liver disease world-wide and can progress to cirrhosis and hepatocellular carcinoma ${ }^{12}$. The global prevalence of NAFLD is thought to be constantly increasing, being currently estimated to about $25 \%$ of the population ${ }^{13}$. The review by Rada et al. discuss some of the molecular mechanisms that can be responsible for lipotoxicity in NAFLD, including ER and oxidative stress, autophagy, lipoapoptosis, and inflammation. In particular, the review highlights the role of CD36/FAT fatty acid translocase in NAFLD pathogenesis. Clinical studies have shown that CD36 increased levels in the liver of NAFLD patients as well as circulating levels of a soluble form of CD36 (sCD36) that are positively correlated with the histological grade of hepatic steatosis. The authors highlight how CD36 or some of its functional regulators may be a promising therapeutic approach for the prevention and treatment of NAFLD and consequently of cirrhosis and hepatocellular carcinoma. We hope that the readers enjoy this review set which represents just the tip of the iceberg of our past, present, and future commitment to advance translational science. The Cell Death $\mathcal{E}$ Disease foremost goal is to publish a peer-reviewed scientific journal of the highest quality focused on understanding the mechanistic bases of disease. If we achieve this goal, the merit goes to the authors who have contributed their original work to our Journal and to the Editors and Reviewers who provided the expertise for processing the thousand manuscripts we annually receive. We finally would like to thank all the past and present members of our Editorial Office as well as the hard-working production Editors at Springer/ Nature, as they finalize the publication of the high-quality papers to which we became accustomed.

\section{Author details}

'Department of Biology, University of Rome "Tor Vergata", Rome, Italy. ${ }^{2}$ National Institute for infectious Disease IRCCS"Lazzaro Spallanzani", Rome, Italy. ${ }^{3}$ CAS Key Laboratory of Tissue Microenvironment and Tumor, Shanghai Institute of Nutrition and Health, Chinese Academy of Sciences, Shanghai,
China. ${ }^{4}$ The First Affiliated Hospital of Soochow University and State Key Laboratory of Radiation Medicine and Protection, Institutes for Translational Medicine, Soochow University Medical College, Suzhou, China. ${ }^{5}$ Institute of Pharmacology, University of Bern, Bern, Switzerland. ${ }^{6}$ Department of Clinical Immunology and Allergology, Sechenov University, Moscow, Russia

\section{Conflict of interest}

The authors declare that they have no conflict of interest.

\section{Publisher's note}

Springer Nature remains neutral with regard to jurisdictional claims in published maps and institutional affiliations.

Received: 23 November 2020 Accepted: 23 November 2020

Published online: 12 December 2020

\section{References}

1. Kapoor, I., Bodo, J., Hill, B. T., Hsi, E. D. \& Almasan, A. Targeting BCL-2 in B-cell malignancies and overcoming therapeutic resistance. Cell Death Dis. 11, 941 (2020).

2. Cerezo, M. \& Rocchi, S. Cancer cell metabolic reprogramming: a keystone for the response to immunotherapy. Cell Death Dis. 11, 964 (2020).

3. Dong, X. et al. Exosomes and breast cancer drug resistance. Cell Death Dis. 11, 987 (2020).

4. Liu, L., Zhang, Y. \& Lu, J. The roles of long noncoding RNAs in breast cancer metastasis. Cell Death Dis. 11, 749 (2020).

5. Rada, P., González-Rodríguez, Á., García-Monzón, C. \& Valverde, A. M. Understanding lipotoxicity in NAFLD pathogenesis: is CD36 a key driver? Cell Death Dis. 11, 802 (2020).

6. Senichkin, V. V., Streletskaia, A. Y., Gorbunova, A. S., Zhivotovsky, B. \& Kopeina, G. S. Saga of Mcl-1: regulation from transcription to degradation. Cell Death Differ. 27, 405 (2020).

7. Kesh, K. et al. Stroma secreted IL6 selects for "stem-like" population and alters pancreatic tumor microenvironment by reprogramming metabolic pathways. Cell Death Dis. 11, 967 (2020).

8. Shahbandi, A. et al. BH3 mimetics selectively eliminate chemotherapy-induced senescent cells and improve response in TP53 wild-type breast cancer. Cell Death Differ. 27, 3097 (2020).

9. Tito, C. et al. LINCO0174 is a novel prognostic factor in thymic epithelial tumors involved in cell migration and lipid metabolism. Cell Death Dis. 11, 959 (2020).

10. Zhu, Y. et al. LncRNA NEAT1 remodels chromatin to promote the 5-Fu resistance by maintaining colorectal cancer stemness. Cell Death Dis. 11, 962 (2020).

11. Zhang, M. et al. ZEB1-activated LINC01123 accelerates the malignancy in lung adenocarcinoma through NOTCH signaling pathway. Cell Death Dis. 11, 981 (2020).

12. Wang, G. et al. Oncogenic driver genes and tumor microenvironment determine the type of liver cancer. Cell Death Dis. 11, 313 (2020).

13. $\mathrm{Wu}, \mathrm{Z}$. et al. Tumor suppressor ZHX2 inhibits NAFLD-HCC progression via blocking LPL-mediated lipid uptake. Cell Death Differ. 27, 1693 (2020). 